\title{
WELL-BEHAVED NONLINEAR EVOLUTION EQUATION FOR STEEPEST-ENTROPY-ASCENT DISSIPATIVE QUANTUM DYNAMICS
}

\author{
GIAN PAOLO BERETTA \\ Università di Brescia, via Branze 38, 25123 Brescia, Italy \\ beretta@unibs.it \\ www.quantumthermodynamics.org
}

\begin{abstract}
In this paper, we outline the main features of the nonlinear quantum evolution equation proposed by the present author. Such an equation may be used as a model of reduced subsystem dynamics to complement various historical and contemporary efforts to extend linear Markovian theories of dissipative phenomena and relaxation based on master equations, Lindblad and Langevin equations, to the nonlinear and far nonequilibrium domain. It may also be used as the fundamental dynamical principle in theories that attempt to unite mechanics and thermodynamics, such as the Hatsopoulos-Gyftopoulos unified theory which motivated the original development of this well-behaved general nonlinear equation for the evolution of the density operator capable of generating irreversible deterministic relaxation to thermodynamic equilibrium from any far nonequilibrium state even for an isolated system.
\end{abstract}

Keywords: Nonlinear quantum dynamics; entropy and irreversibility; quantum thermodynamics.

\section{Introduction}

Much work has appeared in recent years on the study of entropy-generating irreversible nonequilibrium dynamics. Limited discussions of previous work is available, ${ }^{1-4}$ but no thorough critical review of the subject is available, although it would be very helpful to provide proper acknowledgement of pioneering work, ${ }^{6-11, \text { a }}$ avoid "rediscoveries" ${ }^{5}$ and outline the different frameworks, motivations, approaches, and controversial and interpretational aspects.

To be sure, recent discussions ${ }^{2,5,12, \mathrm{~b}}$ on possible fundamental tests of standard unitary quantum mechanics, related to the existence of "spontaneous decoherence" at the microscopic level, and on understanding and predicting decoherence in important future applications ${ }^{c}$ involving nanometric devices, fast switching times, clock synchronization, superdense coding, quantum computation, teleportation, quantum

\footnotetext{
a Available upon email request to the author or at www.quantumthermodynamics.org.

${ }^{\mathrm{b}}$ See e.g. the references by Czachor et al. cited in Ref. 6 of Ref. 18 .

${ }^{\mathrm{c}}$ See the references by Weinberg, Stern, Aharonov, Imry, Ekert, Holyst, Turski, Vidal, Werner, Unruh, Wald, Bennett, Grigorescu, Miranowicz, Matsueda, and others cited in Ref. 7 of Ref. 18.
} 
cryptography, etc. show that the subject of irreversible nonequilibrium dynamics is by no means settled.

\section{General Class of Nonlinear Evolution Equations for Non-Lindblad Dissipative Quantum Dynamics}

Let $\mathcal{H}(\operatorname{dim} \mathcal{H} \leq \infty)$ be the Hilbert space and $H$ the Hamiltonian operator associated with a system in standard quantum mechanics. We assume that the quantum states are one-to-one with the linear hermitian operators $\rho$ on $\mathcal{H}$ with $\operatorname{Tr}(\rho)=1$ and $\rho \geq \rho^{2}$, and we assume a dynamical equation of the form ${ }^{13-19, a, d}$

$$
\frac{d \rho}{d t}=\rho E(\rho, t)+E^{\dagger}(\rho, t) \rho,
$$

where $E(\rho, t)$ is an operator-valued function of $\rho$ and time that we may call the "evolution" operator and in general is non-hermitian. Without loss of generality, we write $E=E_{+}+i E_{-}$where $E_{+}=\left(E+E^{\dagger}\right) / 2$ and $E_{-}=\left(E-E^{\dagger}\right) / 2 i$ are hermitian operators that, for convenience, we rename as $\Delta M(\rho, t) / 2 k_{\mathrm{B}} \tau(\rho, t)$ and $H(\rho, t) / \hbar$, respectively, so that Eq. (1) takes the form ${ }^{18,19}$

$$
\frac{d \rho}{d t}=-\frac{i}{\hbar}[H(\rho, t), \rho]+\frac{1}{2 k_{\mathrm{B}} \tau(\rho, t)}\{\Delta M(\rho, t), \rho\},
$$

where $[\cdot, \cdot]$ and $\{\cdot, \cdot\}$ are the usual commutator and anticommutator, $H(\rho, t)$ may be assumed independent of $\rho$ (but not necessarily independent of time $t$ ) and identified with the Hamiltonian operator, $\hbar$ is the reduced Planck constant, $k_{\mathrm{B}}$ the Boltzmann constant; moreover, $\Delta M(\rho, t)$ is a hermitian operator-valued, possibly nonlinear function of $\rho$ and time which, together with the positive definite, possibly nonlinear functional $\tau(\rho, t)$, describes the dissipative dynamics of the system, and is such that $\operatorname{Tr}[\rho \Delta M(\rho, t)]=0$ as required to preserve $\rho$ unit trace at all times.

The reason for considering a dynamical law of the form (2) is that the explicit expression of $\Delta M(\rho, t)$ that generates steepest-entropy-ascent (maximal entropy generation) conservative dynamics of an adiabatic system compatible with all thermodynamics requirements ${ }^{4}$ is known. ${ }^{13-19, a, d}$ The steepest-entropy-ascent form of the operator function $\Delta M(\rho)$ is defined in Sec. 3 in terms of the operator ${ }^{17-19}$ $M(\rho, t)=S(\rho)-H(t) / \theta(\rho, t)+\boldsymbol{\mu}(\rho, t) \cdot \mathbf{N} / \theta(\rho, t)$, where $S(\rho)$ is the entropy operator (definition below), and the functionals $\theta(\rho, t)$ and $\boldsymbol{\mu}(\rho, t)$ are defined in Sec. 3, Eqs. (3)-(5). We call operator $M(\rho, t)$ the generalized nonequilibrium Massieufunction operator, because at thermodynamic equilibrium its mean value belongs to the family of entropic characteristic functions introduced by Massieu, ${ }^{20}$ i.e. $\langle M\rangle_{\mathrm{e}}=\langle S\rangle_{\mathrm{e}}-\langle H\rangle_{\mathrm{e}} / T+\boldsymbol{\mu} \cdot\langle\mathbf{N}\rangle_{\mathrm{e}} / T$ where $\langle S\rangle_{\mathrm{e}},\langle H\rangle_{\mathrm{e}},\langle\mathbf{N}\rangle_{\mathrm{e}}, T$ and $\boldsymbol{\mu}$ are the equilibrium entropy, energy, amounts of constituents, temperature and chemical potentials, respectively.

\footnotetext{
${ }^{\mathrm{d}}$ See the references by the present author cited in Ref. 19 of Ref. $18{ }^{\mathrm{a}}$
} 
We consider the space $\mathcal{L}(\mathcal{H})$ of linear operators on $\mathcal{H}$ equipped with the real scalar product $(F \mid G)=\operatorname{Tr}\left(F^{\dagger} G+G^{\dagger} F\right) / 2=(G \mid F)$, so that for any (timeindependent) hermitian $F$ in $\mathcal{L}(\mathcal{H})$ the corresponding mean-value state functional can be written as $\langle F\rangle=\operatorname{Tr}(\rho F)=\operatorname{Tr}(\sqrt{\rho} F \sqrt{\rho})=(\sqrt{\rho} \mid \sqrt{\rho} F)$, and can be viewed as a functional of $\sqrt{\rho}$, the square-root density operator, obtained from the spectral expansion of $\rho$ by substituting its eigenvalues with their positive square roots.

For the evolution equation to be well defined, the functional $\operatorname{Tr}(\rho I)$ where $I$ is the identity on $\mathcal{H}$ must remain equal to unity at all times; therefore, $d \operatorname{Tr}(\rho I) / d t=$ $2(\sqrt{\rho} I \mid \sqrt{\rho} E(\rho, t))=0$, implies the condition $(\sqrt{\rho} \mid \sqrt{\rho} \Delta M(\rho, t))=0$.

For $F$ and $G$ hermitian in $\mathcal{L}(\mathcal{H})$, we introduce the following notation: $\Delta F=F-\operatorname{Tr}(\rho F) I,\langle\Delta F \Delta G\rangle=(\sqrt{\rho} \Delta F \mid \sqrt{\rho} \Delta G)=\operatorname{Tr}(\rho\{\Delta F, \Delta G\}) / 2, \Delta_{F}=$ $\sqrt{\langle\Delta F \Delta F\rangle},\langle[F, G] / 2 i\rangle=\operatorname{Tr}(\rho[F, G]) / 2 i=\langle[F, G] / 2 i\rangle^{*}=-\langle[G, F] / 2 i\rangle$. The rate of change of the mean value of a time-independent observable $F$ is $\mathrm{d} \operatorname{Tr}(\rho F) / \mathrm{d} t=$ $2(\sqrt{\rho} F \mid \sqrt{\rho} E(\rho, t))=2\langle[F, H(t)] / 2 i\rangle / \hbar+\langle\Delta F \Delta M(\rho, t)\rangle / k_{\mathrm{B}} \tau$ from which we see that not all operators $F$ that commute with $H(t)$ correspond to constants of the motion, but only those for which $\langle\Delta F \Delta M(\rho, t)\rangle=0$, i.e. such that $\sqrt{\rho} \Delta F$ is orthogonal to both $i \sqrt{\rho} \Delta H(t)$ and $\sqrt{\rho} \Delta M(\rho, t)$, in the sense of the scalar product defined above. For an isolated system, conservation of the mean energy functional $\operatorname{Tr}(\rho H(t))$ requires an operator function $\Delta M(\rho, t)$ that maintains $\sqrt{\rho} \Delta M(\rho, t)$ always orthogonal to $\sqrt{\rho} \Delta H(t)$, so that $\langle\Delta H \Delta M(\rho, t)\rangle=0$ for every $\rho$.

We define the entropy operator $S=-k_{\mathrm{B}} P_{\rho>0} \ln \rho$, where $P_{\rho>0}$ is the projection operator onto the range of $\rho$. ${ }^{\text {e }}$ The entropy functional is $\langle S\rangle=\operatorname{Tr}(\rho S)=$ $-k_{\mathrm{B}} \operatorname{Tr}(\rho \ln \rho)=-2 k_{\mathrm{B}}(\sqrt{\rho} \mid \sqrt{\rho} \ln \sqrt{\rho})$ and its rate of change using Eq. (2) may be written as $d \operatorname{Tr}(\rho S) / d t=2(\sqrt{\rho} S \mid \sqrt{\rho} E(\rho, t))=\langle\Delta S \Delta M(\rho, t)\rangle / k_{\mathrm{B}} \tau(\rho, t)$. Interestingly, the rate of entropy change, being proportional to the correlation coefficient between entropy measurements and $M$ measurements, under the assumptions made so far, may be positive or negative, depending on how $M$ is defined, i.e. depending on the specifics of the physical model in which Eq. (2) is adopted.

\section{Conservative Steepest-Entropy-Ascent Dynamics for an Isolated System}

We now further assume the explicit form of $\Delta M(\rho)$ that yields steepest-entropyascent, conservative dissipative dynamics ${ }^{13,17-19}$

$$
\Delta M(\rho, t)=\Delta S-\Delta H(t) / \theta(\rho, t)+\boldsymbol{\mu}(\rho, t) \cdot \Delta \mathbf{N} / \theta(\rho, t),
$$

where $S$ is the entropy operator defined above, $H(t)$ is the Hamiltonian, $\mathbf{N}=$ $\left\{N_{1}, \ldots, N_{r}\right\}$ a (possibly empty) set of operators commuting with $H$, that we call non-Hamiltonian generators of the motion (for example, the number-of-particles

\footnotetext{
e Operator $S$, first introduced in Refs. 13, 14 and by the present author cited in Ref. 19 of Ref. 18, is always well defined for any $\rho \geq \rho^{2}$. It is the null operator when $\rho^{2}=\rho$. In general, $\sqrt{\rho} S=$ $-k_{\mathrm{B}} \sqrt{\rho} \ln \rho$.
} 
operators or a subset of them, or the momentum component operators for a free particle), such that operators $\sqrt{\rho} \Delta H(t)$ and $\sqrt{\rho} \Delta \mathbf{N}$ are linearly independent, $\theta(\rho, t)$ and $\boldsymbol{\mu}(\rho, t)=\left\{\mu_{1}(\rho, t), \ldots, \mu_{r}(\rho, t)\right\}$ a set of real functionals defined for each $\rho$ by the solution of the following system of linear equations:

$$
\begin{aligned}
\langle\Delta S \Delta H\rangle \theta+\sum_{i}\left\langle\Delta N_{i} \Delta H\right\rangle \mu_{i} & =\langle\Delta H \Delta H\rangle, \\
\left\langle\Delta S \Delta N_{j}\right\rangle \theta+\sum_{i}\left\langle\Delta N_{i} \Delta N_{j}\right\rangle \mu_{i} & =\left\langle\Delta H \Delta N_{j}\right\rangle,
\end{aligned}
$$

which warrant the conditions that $\langle\Delta H \Delta M\rangle=0$ and $\left\langle\Delta N_{j} \Delta M\right\rangle=0$, and hence that the mean values $\operatorname{Tr}(\rho H)$ and $\operatorname{Tr}(\rho \mathbf{N})$ are maintained time invariant by the dissipative term of the equation of the motion. For explicit expressions of $\theta(\rho)$ and $\mu_{i}(\rho)$ see Refs. 14 and 17.

Operators $\sqrt{\rho} \Delta M$ and $\sqrt{\rho} \Delta H^{\prime}=\sqrt{\rho}[\Delta H-\boldsymbol{\mu}(\rho, t) \cdot \Delta \mathbf{N}]$ are always orthogonal to each other, in the sense that $\left\langle\Delta M \Delta H^{\prime}\right\rangle=0$ for every $\rho$. It follows that, in general, $\theta=\left\langle\Delta H^{\prime} \Delta H^{\prime}\right\rangle /\left\langle\Delta S \Delta H^{\prime}\right\rangle,\langle\Delta S \Delta M\rangle=\langle\Delta M \Delta M\rangle=\langle\Delta S \Delta S\rangle-\left\langle\Delta H^{\prime} \Delta H^{\prime}\right\rangle / \theta^{2} \geq 0$, and hence the rate of entropy generation is always strictly positive except for $\langle\Delta M \Delta M\rangle=0$ (which occurs if and only if $\sqrt{\rho} \Delta M=0$ ), i.e. for $\sqrt{\rho_{\text {nd }}} \Delta S_{\text {nd }}=$ $\left(\sqrt{\rho_{\mathrm{nd}}} \Delta H(t)-\boldsymbol{\mu}_{\mathrm{nd}} \cdot \sqrt{\rho_{\mathrm{nd}}} \Delta \mathbf{N}\right) / \theta_{\text {nd }}$, for some real scalars $\theta_{\text {nd }}$ and $\boldsymbol{\mu}_{\text {nd }}$. The nondissipative density operators take the form

$$
\rho_{\mathrm{nd}}=\frac{B(t) \exp \left[-\left(H(t)-\boldsymbol{\mu}_{\mathrm{nd}} \cdot \mathbf{N}\right) / k_{\mathrm{B}} \theta_{\mathrm{nd}}\right] B(t)}{\operatorname{Tr} B(t) \exp \left[-\left(H(t)-\boldsymbol{\mu}_{\mathrm{nd}} \cdot \mathbf{N}\right) / k_{\mathrm{B}} \theta_{\mathrm{nd}}\right]},
$$

where $B(t)$ is a projection operator on $\mathcal{H}\left(B^{2}=B\right)$ evolving unitarily according to $d B / d t=-i[H(t), B] / \hbar$.

The functional $\theta(\rho)$ may be interpreted in this framework as a natural generalization to nonequilibrium of the temperature, at least insofar as for $t \rightarrow+\infty$, while the state operator $\rho(t)$ approaches a non-dissipative operator of form (6), $\theta(t)$ approaches smoothly the corresponding thermodynamic equilibrium (or partial equilibrium) temperature $\theta_{\text {nd }}$.

Because here $H(t)$ always commutes with $M(\rho, t), \sqrt{\rho} \Delta M(\rho, t)$ is always orthogonal to $i \sqrt{\rho} \Delta H(t)$. This reflects the fact that on the entropy surface the direction of steepest entropy ascent is orthogonal to the (constant entropy) orbits that characterize purely Hamiltonian (unitary) motion (in which the entropy is maintained constant by keeping invariant each eigenvalue of $\rho$ ).

As shown in references by the present author cited in Ref. 19 of Ref. $18^{\mathrm{a}}$, the dissipative dynamics generated by Eq. (2) with a time-independent Hamiltonian $H$ and $\Delta M(\rho)$ as just defined: (i) maintains $\rho(t) \geq \rho^{2}(t)$ at all times, both forward and backward in time for any initial density operator $\rho(0)$ (see also Refs. 5 and 17); (ii) maintains the cardinality of $\rho(t)$ invariant; (iii) entails that the entropy functional is an $S$-function in the sense defined in Ref. 21 and therefore that maximal entropy density operators obtained from (6) with $B=I$ are the only equilibrium states of the dynamics that are stable with respect to perturbations that do not alter the mean values of the energy and the other time invariants (if any): this theorem of 
the dynamics coincides with the well-known Hatsopoulos-Keenan general statement of the second law of thermodynamics ${ }^{8,22}$; (iv) entails Onsager reciprocity in the sense defined in Ref. 16; (v) can be derived from a variational principle,,$^{5,17}$ equivalent to our steepest entropy ascent geometrical construction, ${ }^{19, \mathrm{~d}}$ by maximizing the entropy generation rate subject to the $\operatorname{Tr}(\rho), \operatorname{Tr}(\rho H)$, and $\operatorname{Tr}(\rho \mathbf{N})$ conservation constraints and the additional constraint $(\sqrt{\rho} E \mid \sqrt{\rho} E)=c(\rho) \cdot{ }^{17,19}$

We finally note that assuming in Eq. (2), in addition to $\Delta M(\rho)$ given by (3)-(5), the nonlinear relaxation time $\tau(\rho)$ given by ${ }^{17,19} \tau(\rho)=\hbar \Delta_{M} / 2 k_{\mathrm{B}} \Delta_{H}$, we obtain the most dissipative (maximal entropy generation rate $^{17}$ ) dynamics in which the entropic characteristic time ${ }^{19} \tau_{S}=\Delta_{S} /|\mathrm{d}\langle S\rangle / \mathrm{d} t|$ is always compatible with the time-energy uncertainty relation $\tau_{S} \Delta_{H} \geq \hbar / 2$ and the rate of entropy generation is always given by $2 \Delta_{M} \Delta_{H} / \hbar$.

The usefulness and physical meaning of the proposed nonlinear model equation is worth further investigations and experimental validation in specific far nonequilibrium contexts in which linear models of Lindblad form are insufficient. One such context may be the currently debated so-called "fluctuation theorems" ${ }^{23-26}$ whereby fluctuations and, hence, uncertainties are measured on a microscopic system (optically trapped colloidal particle, ${ }^{27,28}$ electrical resistor ${ }^{29}$ ) driven at steady state (off thermodynamic equilibrium) by means of a work interaction, while a heat interaction (with a bath) removes the entropy being generated by irreversibility. Another such context may be that of pion-nucleus scattering, where available experimental data have recently allowed partial validation ${ }^{30}$ of "entropic" uncertainty relations. ${ }^{31-33}$ Yet another is within the model we propose in Ref. 18 for the description of the irreversible time evolution of a perturbed, isolated, physical system during relaxation toward thermodynamic equilibrium by spontaneous internal rearrangement of the occupation probabilities, rather than by interactions with a thermal bath.

\section{MIT School Unified Theory of Mechanics and Thermodynamics}

Thanks to impressive technological advances during the last decades in the manipulation of smaller and smaller systems, down to the single atom scale, the laws of thermodynamics, that fifty years ago were invariably understood as pertaining only to macroscopic phenomena have gradually earned a central role also in studies of mesoscopic phenomena first, and more recently even microscopic phenomena. A new important convergence seems to be emerging around the idea that physical entropy may be a microscopic quantity and that irreversibility should be incorporated in the microscopic description. The differences of interpretation and the various schools of thought still make this subject very controversial. But there is no doubt that credit for this fundamental ansatz, as well as for including it in a coherent and complete new quantum theory that goes beyond quantum mechanics without contradicting it, by incorporating equilibrium and nonequilibrium thermodynamics, must be given to the pioneering contributions of the MIT school. ${ }^{3,8,14,15,34, \mathrm{a}, \mathrm{d}}$ 
The fundamental observation of this school is that the postulates of quantum mechanics should be supplemented with the principles of thermodynamics by assuming a state domain broader than that of the pure states assumed in standard QM. It has been first proposed by Hatsopoulos and Gyftopoulos ${ }^{8}$ on physical and theoretical reasoning only, ${ }^{35,36}$ that in addition to the pure states of standard QM there are states (true states, not heterogeneous preparations ${ }^{13}$ ) that need to be represented by nonidempotent density operators $\rho^{2} \neq \rho$. The entropy functional $-k_{\mathrm{B}} \operatorname{Tr} \rho \ln \rho$ is a measure of the nonidempotence of these states, and emerges from the theory as an intrinsic property of matter, the physical entropy of thermodynamics, ${ }^{37}$ that can be assigned to each and every individual member system of an homogeneous ensemble. The present author added ${ }^{13}$ an equation of motion to complete this theory with a dynamical law that extends the Schrödinger equation to the domain of nonidempotent density operators.

Thirty years ago, the hypothesis of a state domain augmented with respect to that of traditional QM was perceived as adventurous ${ }^{38, a}$ and countercurrent to the prevailing understanding of dissipative quantum dynamics within the frameworks of statistical, stochastic, phenomenological, information-theoretic, chaotic-behavior and bifurcation theories.

The recent advances and the impressive effort devoted to study nonlinear modifications of the standard Schrödinger equation in the last twenty years, finally seem to make more acceptable, ${ }^{5}$ if not require, the $\rho^{2} \neq \rho$ augmented state domain Hatsopoulos-Gyftopoulos (HG) ansatz. ${ }^{39}$

Once the $\rho^{2} \neq \rho$ ansatz is accepted, the nonlinear equation of motion we proposed completes the dynamics and holds the promise to provide a microscopic-level explanation of the recent experimental evidence of spontaneous losses of quantum coherence. It is with this motivation that Gheorghiu-Svirschevski ${ }^{2,5}$ has "rediscovered" our equation of motion. However, in Ref. 5, the question of defining the form of the relaxation-time functional is left unresolved, together with the nontrivial problem to extend the nonlinear dynamics to composites of possibly interacting and entangled subsystems, that we address in Refs. 15 and $17 .^{\text {a }}$

\section{References}

1. M. Lemanska and Z. Jaeger, Physica D 170 (2002) 72.

2. S. Gheorghiu-Svirschevski, Phys. Rev. A 63 (2001) 054102.

3. R. F. Simmons and J. L. Park, Found. Phys. 11 (1981) 297.

4. G. P. Beretta, Mod. Phys. Lett. A 20 (2005) 977.

5. S. Gheorghiu-Svirschevski, Phys. Rev. A 63 (2001) 022105.

6. J. L. Park, Found. Phys. 21 (1991) 83.

7. J. L. Park and W. Band, Found. Phys. 22 (1992) 657.

8. G. N. Hatsopoulos and E. P. Gyftopoulos, Found. Phys. 6 (1976) 15.

9. G. N. Hatsopoulos and E. P. Gyftopoulos, Found. Phys. 6 (1976) 127.

10. G. N. Hatsopoulos and E. P. Gyftopoulos, Found. Phys. 6 (1976) 439.

11. G. N. Hatsopoulos and E. P. Gyftopoulos, Found. Phys. 6 (1976) 561.

12. G. Domokos and S. Kovesi-Domokos, J. Phys. A 32 (1999) 4105. 
13. G. P. Beretta, On the general equation of motion of quantum thermodynamics and the distinction between quantal and nonquantal uncertainties, Sc. D. Thesis, M.I.T. (1981) [quant-ph/0509116].

14. G. P. Beretta, E. P. Gyftopoulos, J. L. Park and G. N. Hatsopoulos, Nuovo Cimento B 82 (1984) 169.

15. G. P. Beretta, E. P. Gyftopoulos and J. L. Park, Nuovo Cimento B 87 (1985) 77. ${ }^{\text {a }}$

16. G. P. Beretta, Found. Phys. 17 (1987) 365. ${ }^{\mathrm{a}}$

17. G. P. Beretta, quant-ph/0112046.

18. G. P. Beretta, Phys. Rev. E 73 (2006) 026113.

19. G. P. Beretta, quant-ph/0511091.

20. M. F. Massieu, Comptes Rendus Acad. Sci. 69 (1859) 858.

21. G. P. Beretta, J. Math. Phys. 27 (1986) $305 .^{\text {a }}$

22. E. P. Gyftopoulos and G. P. Beretta, Thermodynamics: Foundations and Applications (Dover Publications, Mineola, NY, 2005).

23. D. J. Evans and D. J. Searles, Phys. Rev. E 50 (1994) 1645.

24. G. E. Crooks, Phys. Rev. E 60 (1999) 2721.

25. R. Dewar, J. Phys. A 36 (2003) 631.

26. R. van Zon and E. G. D. Cohen, Phys. Rev. Lett 91 (2003) 110601.

27. G. M. Wang et al., Phys. Rev. Lett. 89 (2002) 050601.

28. T. M. Nieuwenhuizen and A. E. Allahverdyan, cond-mat/0207587.

29. N. Garnier and S. Ciliberto, Phys. Rev. E 71 (2005) 060101.

30. D. B. Ion and M. L. D. Ion, Phys. Rev. Lett 81 (1998) 5714.

31. D. Deutsch, Phys. Rev. Lett. 50 (1983) 631.

32. M. H. Partovi, Phys. Rev. Lett 50 (1983) 1883.

33. H. Maassen and J. B. M. Uffink, Phys. Rev. Lett 60 (1988) 1103.

34. J. L. Park, Am. J. Phys. 36 (1968) 211. ${ }^{\mathrm{a}}$

35. J. L. Park, Found. Phys. 18 (1988) 225-244.

36. E. P. Gyftopoulos, Journal of energy resources technology, Trans. ASME 123 (2001) 110.

37. E. P. Gyftopoulos and E. Çubukçu, Phys. Rev. E 55 (1997) 3851.

38. J. Maddox, Nature 316 (1985) $11 .^{\mathrm{a}}$

39. G. P. Beretta, Mod. Phys. Lett. A 21 (2006) 2799. 